\title{
PREDICCIÓN DE LAS RESPUESTAS PSICOLÓGICAS DEL DUELO ANTE LA PÉRDIDA DE SALUD POR VARIABLES DE PERSONALIDAD, RELIGIOSIDAD SOCIODEMOGRÁFICAS, Y EXISTENCIALES
}

\author{
PREDICTION OF PSYCHOLOGICAL RESPONSES OF MOURNING TO LOSS OF HEALTH BY \\ PERSONALITY VARIABLES, SOCIODEMOGRAPHIC RELIGIOSITY, AND EXISTENTIAL \\ DOI: 10.22199/S07187475.2014.0003.00002 \\ Recibido: 15 de Octubre del 2014 | Aceptado: 12 de Noviembre del 2014
}

MELINA MIAJA ÁVILA ${ }_{1}$;OSÉ MORAL DE LA RUBIA 2

(UNIVERSIDAD AUTÓNOMA DE NUEVO LEÓN, Nuevo León, México)

\begin{abstract}
RESUMEN
Objetivo: estudiar la asociación y predecir las respuestas psicológicas del duelo ante la pérdida de la salud por medio de alexitimia, resiliencia, religiosidad y variables sociodemográficas, existenciales y clínicas. Método: se aplicó un cuestionario de preguntas cerradas y abiertas, la escala de respuestas psicológicas del duelo ante la pérdida de la salud, la escala mexicana de resiliencia y la escala de alexitimia de Toronto a una muestra no probabilística de 120 mujeres mexicanas tratadas de cáncer. Resultados: las variables de personalidad de alexitimia y resiliencia, las variables sociodemográficas de ingreso económico y escolaridad, la variable religiosidad, la variable existencial de sentido de la vida y las variables clínicas de efectos secundarios, información, percepción del efecto curativo y adherencia al tratamiento se asociaron con las respuestas psicológicas del duelo. Se especificó un modelo que mostró buen ajuste por Máxima Verosimilitud. En este modelo, bajo afecto negativo fue predicho por menor alexitimia, mayor apoyo familiar, mayor estructura y menores efectos secundarios del tratamiento; la actitud positiva fue predicha por mayor estructura y menor ingreso económico. Conclusión: las dos variables de personalidad estudiadas tuvieron un efecto significativo y mayor que las variables sociodemográficas, de religiosidad, existenciales y clínicas.
\end{abstract}

PALABRAS CLAVE: Cáncer, duelo, alexitimia, resiliencia.

\section{ABSTRACT}

Objective: To study the associations and predict the psychological answers to mourning when facing health deterioration caused by personality, religiosity, sociodemographic, existential, and clinical variables. Method: A questionnaire of closed and open questions, the scale of psychological answers to mourning when facing health deterioration, the Mexican scale of resilience and the scale of alexithymia from Toronto were applied to a non-probabilistic sample of 120 Mexican female cancer patients. Results: All variables were associated with the psychological answers to mourning. A model showing a good adjustment to Maximum Verisimilitude was specified. This model, under negative effects, was predicted by less alexithymia, more family support, a higher structure, and less treatment secondary effects. Positive attitude was predicted by a higher structure and a less economic income. Conclusion: The two personality variables had a significant and major effect than the sociodemographic, existential, and clinical variables and the variables of religiosity.

KEY WORDS: Cancer, mourning, alexithymia, resilience. 
Las personas que tienen enfermedades crónicas pasan por un proceso conocido como duelo ante la pérdida de la salud (Hirsch, 2010). Uno de los modelos que describen la evolución del duelo más conocido es el de Kübler-Ross. Según este modelo, las personas que se encuentran próximas a la muerte transitan por una secuencia de cinco fases: negación, ira, negociación/pacto, depresión y aceptación. Dichas fases tienen una duración variable. Usualmente se suceden unas a otras, aunque puede haber solapamientos $y$ fluctuaciones en esta secuencia (KüblerRoss, 2005).

La aceptación de una enfermedad crónica se ha relacionado con mayor resiliencia (Bonanno, 2004), mayor apoyo familiar y social (Pentz, 2005), así como mayor espiritualidad y tendencia a dar nuevos significados a la vida ante eventos que la comprometen (Büssing \& Koenig, 2010). La resiliencia hace referencia a los recursos con los que cuentan las personas para adaptarse a situaciones de estrés intenso, de ahí que las personas con más resiliencia logren desestructurarse menos y acepten antes la situación de pérdida de la salud (Bonanno, 2004). Las creencias religiosas proporcionan una esperanza de curación y facilitan construir un significado positivo a la muerte, aparte el apoyo de la comunidad religiosa en las personas muy practicantes les refuerza estas vías de significación e incluso les puede proporcionar recursos materiales ante la necesidad; de ahí que la religiosidad facilite el proceso de duelo (Bussing \& Koenig, 2010; Peteet \& Balboni, 2013). Un duelo exitoso queda garantizado si la persona logra dar a la situación dolorosa de enfermedad y a la expectativa de muerte un sentido para vivir más intensamente, cerrar asuntos pendientes y prepararse para la transcendencia espiritual; de ahí que un cambio positivo del sentido de la vida se asocie con un duelo exitoso (Worden, 2010).
Thompson et al. (2009) observaron que los pacientes con cáncer con más dificultades para aceptar su enfermedad tenían menor escolaridad, edad y apoyo social. Exline, Park, Smyth y Carey (2011) hallaron que menor religiosidad y menor edad predicen una peor adaptación al cáncer. No obstante, hay estudios que reportan que sexo, edad y duración de la enfermedad son independientes de la aceptación de la enfermedad (Zalewska, Miniszewska, Chodkiewicz, \& Narbutt, 2007). En la medida en que las personas tienen bajos ingresos, usualmente asociados con baja escolaridad, la situación de enfermedad les supone una carga importante, especialmente si no cuentan con seguro de enfermedad, lo que incrementa el afecto negativo asociado con la situación de duelo (Aguilera, Marrufo, \& Montesino, 2005). Además, tener hijos pequeños, usualmente asociado con menor edad en adultos, genera mayor angustia ante la posibilidad de morirse y estar incapacitado (Exline et al., 2011).

Según Kübler-Ross (2005), se va completando el proceso de duelo en la medida que transcurre el tiempo desde el diagnóstico, apareciendo primero la negación e ira. Mayor información sobre la enfermedad puede aminorar la angustia al poder la persona anticipar y prepararse para los cambios esperados (Schlatter \& Cameron, 2010). La percepción de efectos secundarios, la pobre adherencia al tratamiento y la falta de percepción de efectos curativos se interrelacionan e incrementan el afecto negativo, aunque el afecto negativo puede generar un sesgo de distorsión perceptiva y desmotivación (Thompson et al., 2009).

Un resultado bien establecido es que los recursos personales tienen mayor tamaño del efecto sobre las respuestas psicológicas del duelo que las variables sociodemográficas y clínicas (O'Brien \& Moorey, 2010; Van-Damme, Crombez, VanHoudenhove, Mariman \& Michielsen, 2006). Otro hallazgo bien establecido es la 
correlación positiva entre religiosidad y aceptación de la enfermedad (Peteet \& Balboni, 2013).

La alexitimia es un concepto importante dentro de la psicología de la salud que hace referencia al manejo de las emociones, lo que hace a este constructo relevante para el proceso de duelo. La alexitimia es un rasgo de personalidad caracterizado por dificultad para identificar y expresar verbalmente emociones junto con un estilo de pensamiento externamente orientado y pobreza de la fantasía (Taylor \& Bagby, 2013). Los estudios revelan que la alexitimia tiene un efecto de grande a moderado sobre la depresión, la ansiedad y las dificultades de adaptación (Mantani et al, 2007; Rueda \& Pérez, 2007). Lumley (2004) señala que las personas alexitímicas, al no ser capaces de diferenciar y modular su estado emocional negativo, mantienen un nivel permanente de anhedonia y malestar emocional.

Existen estudios del efecto de la alexitimia sobre la depresión y ansiedad ante la pérdida de la salud (Mantani et al., 2007) y sobre la ira (Rueda \& Pérez), pero no se ha aplicado el concepto a todas las respuestas del duelo del modelo de KüblerRoss; a su vez, se desconoce qué influencia tenga la alexitimia sobre las respuestas psicológicas del duelo frente a otros variables significativas, como resiliencia, práctica religiosa, ingreso económico, efectos secundarios y adherencia al tratamiento, así como resignificación de la vida.

Considerando este vacío en las publicaciones, los objetivos de esta investigación son estudiar la asociación y predecir las respuestas psicológicas del duelo ante la pérdida de la salud (negación, ira, fe/esperanza, promesas/negociación, depresión y aceptación) en mujeres con cáncer por medio de las variables de personalidad de alexitimia y resiliencia, las variables de religiosidad de convicción y frecuencia de práctica, las variables sociodemográficas de edad, escolaridad e ingreso económico familiar, las variables existenciales de cambio en la visión de la vida y mayor sentido de la vida a raíz del diagnóstico y las variables clínicas de tiempo transcurrido desde el diagnóstico al momento de la encuesta, información, percepción de efectos curativos, percepción de efectos secundarios y adherencia al tratamiento oncológico.

Debido a los centros a los que se tuvo acceso al recolectar la muestra, hubo muchas dificultades para encuestar a hombres, lo que provocó una desproporción entre ambos sexos en la integración de la muestra, aproximadamente 1 hombre por cada 5 mujeres. Menor alexitimia (Taylor \& Bagby, 2013), mayor propensión a la depresión y la ansiedad (Barreto, de la Torre \& Pérez, 2012), mayor tendencia a buscar el apoyo familiar y social ante situaciones de estrés (Bonanno, 2004; Cardoso, Ramos, Vaz, Rodríguez \& Fernández, 2012; Kübler-Ross, 2005; O'Brien \& Moorey, 2010) y mayor religiosidad (Büssing \& Koenig, 2010) se han reportado en mujeres en comparación con hombres. Considerando estos hallazgos, se optó por reducir la muestra al sexo femenino para evitar la pérdida de potencia al contrastar invarianza 0 diferencias y el sesgo en la muestra conjunta por la composición desproporcionada de ambos sexos.

El marco teórico del presente estudio se asienta en Kübler-Ross (2005) para conceptualizar el duelo y teorías de la personalidad sobre la alexitimia y la resiliencia como factores de riesgo/protección, aparte de variables relevantes en la investigación y abordaje psicológico del paciente terminal, como la religiosidad, aspectos existenciales y sociodemográficos. Una teoría bajo la que se podría enmarcar el planteamiento general de este estudio sería la de estresorafrontamiento/personalidad-respuesta de estrés, siendo un ejemplo de este tipo de 
teorías, la de Grossarth-Maticek y Eysenck (1991).

Se espera que la alexitimia tenga una asociación significativa con las respuestas psicológicas del duelo, especialmente con afecto negativo (Mantani et al., 2007; Rueda \& Pérez, 2007), y que su tamaño del efecto sea mayor que el de la resiliencia (al tratarse de un déficit en recursos básicos), ingreso económico (que suele ser la variable sociodemográfica más asociada con el duelo), práctica religiosa (que suele estar más asociada con el duelo que convicción religiosa) y las variables existenciales; asimismo, se espera interrelación entre los predictores.

\section{MÉTODO}

\section{Participantes}

Los criterios de inclusión fueron: tener enfermedad oncológica diagnosticada, recibir tratamiento oncológico, tener al menos 15 años de edad, saber leer y escribir y firmar el consentimiento informado. El criterio de exclusión fue fatiga excesiva. La muestra se redujo exclusivamente a mujeres debido a que en los centros en los cuales fue recolectada (orientados a la mujer) había muy pocos pacientes masculinos.

La muestra quedó formada por 120 participantes. Se extrajo de siete lugares enfocados al cáncer femenino; 83 de las 120 mujeres $(69 \%)$ fueron entrevistadas en tres albergues, 31 (26\%) en dos clínicas y 6 (5\%) en dos grupos de autoayuda de Monterrey, México. La media de edad fue 51.84 años $(D E=13.63)$. La media de escolaridad correspondió a secundaria terminada. La media de ingreso económico familiar al mes correspondió al intervalo de 6.000 a 11.999 pesos mexicanos (aproximadamente de 500 a 1.000 dólares estadounidenses). Con respecto al estado civil, 76 de las 120 participantes (63\%) dijeron estar casadas, 20 (17\%) solteras, 10 $(8 \%)$ viudas, $8(7 \%)$ separadas y $6(5 \%)$ en unión libre. Con respecto a la adscripción religiosa, 98 de 120 mujeres (82\%) indicaron ser católicas, 15 (12.5\%) cristianas, $5(4.2 \%)$ pertenecer a otra religión distinta de la católica o cristiana y 2 $(1.7 \%)$ a ninguna; $56(46.7 \%)$ dijeron tener total convicción religiosa, $39(32.5 \%)$ bastante, 19 (15.8\%) algo, 4 (3.3\%) poco y $2(1.7 \%)$ ninguna; $66(55 \%)$ indicaron asistir con frecuencia a eventos religiosos, 24 (20\%) con mucha frecuencia, $20(16.7 \%)$ en raras ocasiones aparte de eventos especiales y 10 (8.3\%) sólo a eventos especiales.

La media del tiempo transcurrido desde el diagnóstico de cáncer fue de 1 año y 5 meses, $(D E=2$ años). Respecto al tipo de cáncer, 60 de las 120 mujeres (50\%) indicaron padecer cáncer de mama, 19 (15.8\%) cáncer cervicouterino, 14 (11.7\%) cáncer en el sistema digestivo, 9 (7.5\%) sarcoma, $6(5 \%)$ cáncer de riñón, 4 (3.3\%) cáncer en glándulas endocrinas, $3(2.5 \%)$ linfomas, $2(1.7 \%)$ melanoma, $2(1.7 \%)$ leucemia y $1(0.8 \%)$ cáncer cerebral; 58 $(48.3 \%)$ indicaron recibir radioterapia, 55 $(45.8 \%)$ quimioterapia, $5(4.2 \%)$ cirugía y 2 $(1.7 \%)$ terapia hormonal.

\section{Instrumentos de medida}

Se aplicaron cinco preguntas cerradas sobre datos sociodemográficos (sexo, edad, escolaridad, ingreso económico familiar mensual y afiliación religiosa), dos sobre religiosidad con un rango de 1 a 4 (convicción en las creencias religiosas y frecuencia de asistencia a los servicios religiosos) y dos sobre aspectos vivenciales (¿su forma de ver la vida cambio durante el transcurso de la enfermedad? [rango de 1 a 5], y ¿considera que esta situación, le dio mayor sentido a su vida? [rango de 1 a 3]) y cinco sobre aspectos clínicos (tiempo transcurrido desde el diagnóstico al momento de la encuesta, efectos secundarios del tratamiento [rango de $1 \mathrm{a}$ 3], poseer información sobre la enfermedad, tratamiento y curso clínico [suma de 3 ítems con un rango de 3 a 15], percibir que el tratamiento está teniendo éxito y adherencia 
al tratamiento [ambas con un rango de $1 \mathrm{a}$ 3]).

Escala Mexicana de Resiliencia (RESIM) de Palomar y Gómez (2010). Está conformada por 43 ítems con un rango de 5 puntos (de 1 = "totalmente en desacuerdo" a 5 = "totalmente de acuerdo"). Presentó consistencia interna alta $(\alpha=.93)$ y una estructura de cinco factores: fortaleza y confianza en sí mismo (FC) con 19 ítems (a $=.92$ ), competencia social (CS) con 8 ítems $(a=.87)$, apoyo familiar (AF) con 6 items ( $a$ $=.87$ ), apoyo social (AS) con 5 ítems ( $a=$ .84) y estructura (E) con 5 ítems referentes a la capacidad de las personas para organizarse y planear las actividades aún en momentos difíciles $(\alpha=.79)$ (Palomar \& Gómez, 2010).

Escala de Alexitimia de Toronto de 20 reactivos (TAS-20), usando la adaptación a población mexicana de Moral (2008). Consta de 20 ítems con un rango de 6 puntos (de 0 "totalmente en desacuerdo" a 5 "totalmente de acuerdo"). Su consistencia interna fue alta $(\alpha=.82)$ y mostró estabilidad temporal a los 6 meses $(r=.71)$. Presentó una estructura de tres factores: dificultad para identificar sentimientos (DIS) con 7 indicadores $(a=.78)$, dificultad para expresar verbalmente sentimientos (DES) con 5 indicadores $(\alpha=.80)$ y pensamiento externamente orientado (PEO) con 8 indicadores $(\alpha=.53)$ (Moral, 2008).

La Escala de Respuestas Psicológicas del Duelo ante la Pérdida de la Salud (RPDPS-38) de Miaja y Moral (2014). Está conformada por 38 ítems con un rango de respuesta de cinco puntos (de $1=$ nunca a 5 = siempre). Presentó una estructura de 6 factores de primer orden: negación (Nega) con 7 ítems $(\alpha=.72)$, ira (Ira) con 6 ítems ( $\alpha$ $=.82$ ), promesas/negociación con un Ser Supremo (P/Nego) con 7 ítems $(\alpha=.83)$, fe/esperanza (Fe/Es) en la recuperación con 4 ítems $(\alpha=.80)$, depresión (Depre) con 4 ítems ( $a=.73$ ) y aceptación (Acep) con 10 ítems $(\alpha=.85)$. Se definieron 2 factores de segundo orden 0 dimensiones independientes: afecto negativo (ANE) con consistencia interna alta para los 27 ítems de las 4 escalas que lo definen $(\alpha=.87)$ y actitud positiva ante la enfermedad con apoyo en creencias religiosas (APE) con consistencia interna alta para los 11 ítems de las dos escalas que lo definen $(\alpha=.85)$. Las puntuaciones en los 6 factores de primer se obtienen por suma simple de ítems. La dimensión de afecto negativo se obtiene sumando directos los 17 ítems de depresión, ira y negación y sumando invertidos los 10 ítems de aceptación. La dimensión de actitud positiva se obtiene sumando directos los 11 ítems de promesas/negociación y fe/esperanza (Miaja \& Moral, 2014).

\section{Procedimiento}

El proyecto fue revisado y aprobado en sus aspectos éticos y técnicos por una Comisión de Posgrado de la Facultad de Psicología de la Universidad Autónoma de Nuevo León. Antes de comenzar el estudio se proporcionó el protocolo de investigación a las instituciones anfitrionas para obtener la aprobación por sus autoridades. Se solicitó el consentimiento informado para la participación en el estudio, garantizando el anonimato y confidencialidad de la información de acuerdo con las normas éticas de la American Psychological Association (2002). Para la administración del instrumento se pidió a las pacientes firmar la carta del consentimiento informado. Las participantes que aceptaron formar parte del estudio respondieron por escrito al instrumento en presencia de la entrevistadora (primera autora del artículo), en salas polivalentes, cuartos, pasillos 0 lugares permitidos por cada una de las instituciones. La muestra se recolectó de marzo a octubre del 2013.

\section{Análisis estadísticos}

Aunque existen bases teóricas en el planteamiento de este estudio (conceptualización del duelo bajo las etapas de Kübler-Ross y teoría del estresor [cáncer]-afrontamiento/personalidad 
[alexitimia, resiliencia, religiosidad y recursos materiales]-respuesta de estrés [respuestas de duelo]), la exploración de datos (correlaciones y modelos de regresión) guían la especificación del modelo.

Las relaciones lineales de la alexitimia, resiliencia y variables sociodemográficas, de religiosidad, existenciales y clínicas con las respuestas psicológicas del duelo se calcularon por el coeficiente de correlación producto-momento de Pearson $(r)$ y la correlación canónica $\left(r_{c}\right)$. Las magnitudes de asociación de valores de $r$ y $r_{c}<.30 \mathrm{se}$ consideraron bajas, de .30 a .49 moderadas, de .50 a .69 altas, de .70 a .89 muy altas $\mathrm{y} \geq .90$ casi perfectas.

Para predecir las respuestas psicológicas del duelo se usó regresión lineal múltiple por el método de pasos sucesivos y modelamiento de ecuaciones estructurales por el método de Máxima Verosimilitud (ML). En los modelos de regresión sólo se introdujeron correlatos significativos con la variable-criterio.

Se consideraron diez índices para interpretar el ajuste del modelo a los datos: prueba chi-cuadrado ( $\mathrm{X} 2)$, cociente entre el estadístico chi-cuadrado y sus grados de libertad ( $x 2 / g l)$, probabilidad de Bollen-Stine ( $p$ de B-S), índice de bondad de ajuste (GFI) de Jöreskog y Sörbom y su modalidad corregida (AGFI), índice normado de ajuste (NFI) de Bentler-Bonett, índice comparativo de ajuste (CFI) de Bentler, residuo estandarizado cuadrático medio (RMS SR) de Jöreskog y Sörbom y error de aproximación cuadrático medio (RMSEA) de Steiger-Lind. Se estipularon como valores de buen ajuste: $p$ de $\chi 2$ y $p$ de B-S $>.05$, $x 2 / g l \leq 2$, RMSEA y RMS SR $\leq .05$, GFI $\geq$ .95 y AGFI, NFI y $\mathrm{CFI} \geq .90$; y como valores adecuados: $p$ de $X 2$ y $p$ de B-S $>.01, X 2 / g l$ $\leq 3$, RMSEA y RMS SR $\leq .08, \mathrm{GFI} \geq .85 \mathrm{y}$ AGFI, NFI y $\mathrm{CFI} \geq .80$. El cumplimiento del supuesto de normalidad multivariada se observó desde el valor estandarizado de la curtosis multivariada de Mardia. Al ser su valor mayor que 10 , se empleó complementariamente el método de percentiles libres de sesgo para contrastar la significación de parámetros y la prueba de Bollen-Stine para contrastar el ajuste global del modelo, extrayéndose 2,000 muestras. La parsimonia del modelo se calculó por la razón de parsimonia de James-Mulaik-Brett. Se estimaron los tamaños estandarizados del efecto (total, directo e indirecto) sobre cada variable endógena; el tamaño del efecto de valores menores que .10 se consideró trivial, de .10 a .29 pequeño, de .30 a .49 mediano, de .50 a .69 grande y de .70 o mayor muy grande (Kline, 2010). Los cálculos estadísticos fueron hechos con los programas SPSS16 y AMOS16.

\section{RESULTADOS}

Correlatos y predicción de las respuestas psicológicas del duelo por regresión lineal

De las 17 variables correlacionadas con ANE (véase Tabla 1), 6 resultaron predictores significativos. Debido a problemas de colinealidad se optó por estimar el modelo sin 2 variables (competencia social y apoyo social). Calculado el modelo con 4 variables, todas fueron predictores significativos de ANE: alexitimia (TAS-20), apoyo familiar, estructura y efectos secundarios. El modelo explicó el $47 \%$ de la varianza de ANE (véase Tabla 2).

De las 12 variables correlacionadas con APE (véase Tabla 1), 3 resultaron predictores significativos: ingreso económico familiar, estructura y convicción religiosa. El modelo explicó el $25 \%$ de la varianza de APE (véase Tabla 2).

De las 7 variables correlacionadas con negación (véase Tabla 1), 2 resultaron predictores significativos: alexitimia (TAS20) e ingreso económico familiar. El modelo explicó el $17 \%$ de la varianza de negación (véase Tabla 2).

De las 11 variables correlacionadas con ira (véase Tabla 1), 3 resultaron predictores 
significativos: apoyo familiar, alexitimia (TAS-20) y adherencia al tratamiento. El modelo explicó el $32 \%$ de la varianza de ira (véase Tabla 2).

De las 8 variables correlacionadas con promesas/negociación (véase Tabla 1), 2 resultaron predictores significativos: ingreso económico familiar y convicción religiosa. El modelo explicó el $14 \%$ de la varianza de promesas/negociación (véase Tabla 2).

De las 8 variables correlacionadas con fe/esperanza (véase Tabla 1), 4 resultaron predictores significativos: escolaridad, frecuencia de asistencia a los servicios religiosos, el puntaje total de la escala de resiliencia y sentido de vida. El modelo explicó el $26 \%$ de la varianza de fe/esperanza (véase Tabla 2).

De las 13 variables que correlacionaron con depresión (véase Tabla 1), 4 resultaron predictores significativos: dificultad para identificar sentimientos (DIS), efectos secundarios del tratamiento, frecuencia de asistencia a los servicios religiosos e información. El modelo explicó el $39 \%$ de la varianza de depresión (véase Tabla 2).

De las 12 variables correlacionadas con aceptación (véase Tabla 1), 3 resultaron predictores significativos: el puntaje total de la escala de resiliencia, sentido de vida e información. El modelo explicó el $29 \%$ de la varianza de aceptación (véase Tabla 2).

TABLA 1.

Correlaciones de las respuestas del duelo con alexitimia, resiliencia y variables sociodemográficas, existenciales y clínicas.

\begin{tabular}{|c|c|c|c|c|c|c|c|c|}
\hline \multirow{2}{*}{ Variables } & \multicolumn{8}{|c|}{ Respuestas psicológicas del duelo } \\
\hline & ANE & APE & Nega. & Ira & P/Nego & $\mathrm{Fe} / \mathrm{Es}$ & Depre & Acep. \\
\hline \multicolumn{9}{|l|}{ Alexitimia } \\
\hline TAS-20 & $.47^{\star \star \star}$ & $.23^{*}$ & $.37^{\star \star \star}$ & $.38^{* * *}$ & $.19^{*}$ & .10 & $.50^{\star \star \star}$ & $-.19^{*}$ \\
\hline DIS & $.45^{\star \star *}$ & $.21^{*}$ & $.30^{\star * \star}$ & $.35^{\star \star *}$ & $.21^{*}$ & .13 & $.52^{\star \star *}$ & $-.22^{*}$ \\
\hline DES & $.45^{* * *}$ & 17 & $.33^{\star * *}$ & $.36^{* * *}$ & $.19^{*}$ & .07 & $.47^{\star \star *}$ & $-.20^{*}$ \\
\hline PEO & $.39^{\star * *}$ & 13 & $.38^{\star * *}$ & $.36^{* * *}$ & 14 & .06 & $.39^{\star \star *}$ & -.10 \\
\hline \multicolumn{9}{|l|}{ Resiliencia } \\
\hline RESI & $-.44^{\star * *}$ & $.22^{*}$ & -.05 & $-.33^{* * *}$ & .10 & $.22^{*}$ & $-.25^{\star \star}$ & $.47^{\star \star *}$ \\
\hline FC & $-.39^{\star \star *}$ & $.18^{*}$ & -.04 & $-.26^{\star *}$ & .06 & 16 & $-.23^{*}$ & $.47^{* * *}$ \\
\hline CS & $-.24^{\star *}$ & $.20^{*}$ & .06 & -.17 & .09 & $.18^{*}$ & -.15 & $.33^{\star * *}$ \\
\hline AF & $-.52^{\star \star *}$ & 13 & $-.22^{*}$ & $-.45^{\star \star *}$ & .08 & $.20^{*}$ & $-.31^{\star * *}$ & $.40^{\star * *}$ \\
\hline AS & $-.23^{*}$ & .05 & -.06 & $-.20^{*}$ & .03 & .09 & -.15 & .17 \\
\hline$E$ & $-.34^{\star * *}$ & $.29^{* *}$ & .03 & $-.29^{\star *}$ & .15 & $.29^{* *}$ & -.15 & $.39^{\star * *}$ \\
\hline \multicolumn{9}{|c|}{ Variables sociodemográficas } \\
\hline Edad & .03 & .09 & .04 & -.09 & .12 & .09 & .11 & -.06 \\
\hline Ingreso & $-.22^{*}$ & $-.36^{* * *}$ & $-.31^{* * *}$ & -.14 & $-.31^{* * *}$ & $-.26^{\star \star}$ & $-.22^{*}$ & .11 \\
\hline Escolaridad & -.16 & $-.33^{\star * *}$ & $-.23^{*}$ & -.07 & $-.30^{* * *}$ & $-.29^{* \star}$ & $-.19^{*}$ & .11 \\
\hline \multicolumn{9}{|c|}{ Variables de religiosidad } \\
\hline Convicción & -.07 & $.23^{*}$ & .11 & -.00 & $.20^{*}$ & .17 & -.10 & .08 \\
\hline Práctica & $-.30^{* * *}$ & $.30^{* * *}$ & -.02 & $-.22^{*}$ & $.19^{*}$ & $.34^{\star * \star}$ & $-.23^{*}$ & $.19^{*}$ \\
\hline \multicolumn{9}{|c|}{ Variables existenciales } \\
\hline Cambio & -.13 & -.01 & -.04 & -.07 & -.04 & .02 & -.13 & .17 \\
\hline Sentido & $-.22^{*}$ & $.22^{*}$ & .06 & -.15 & .10 & $.24^{* *}$ & -.13 & $.32^{\star \star \star}$ \\
\hline \multicolumn{9}{|c|}{ Variables clínicas } \\
\hline Tiempo & .09 & .06 & .06 & .11 & .09 & -.06 & .10 & -.02 \\
\hline Efectos & $.25^{* *}$ & .07 & .11 & .12 & .10 & .06 & $.25^{* *}$ & -.14 \\
\hline Información & $-.28^{* *}$ & -.16 & -.11 & -.17 & $-.23^{*}$ & -.11 & $-.27^{\star \star}$ & $.29^{* *}$ \\
\hline Tratamiento & $-.20^{*}$ & -.07 & -.03 & -.17 & -.12 & -.02 & $-.24^{\star *}$ & .09 \\
\hline Adherencia & $-.29^{* *}$ & $.18^{*}$ & -.01 & $-.30^{\star * *}$ & .09 & .15 & -.17 & $.22^{*}$ \\
\hline
\end{tabular}

Significación de la correlación: ${ }^{*} p \leq .05,{ }^{* *} p \leq .01 \mathrm{y}{ }^{* *} p \leq .001$. Respuestas psicológicas del duelo: ANE = Afecto negativo ante la enfermedad. $\mathrm{APE}=$ Actitud positiva ante la enfermedad. Nega $=$ Negación, $\mathrm{P} / \mathrm{Nego}=$ Promesas $/$ negociación, Fe/Es $=$ Fe/esperanza, Depre $=$ Depresión y Acep $=$ Aceptación. Alexitimia: TAS-20 = Puntuación total de la escala TAS-20. DIS $=$ Dificultad para identificar sentimientos. DES $=$ Dificultad para expresar sentimientos. PEO = Pensamiento externamente orientado. Resiliencia: RESI = Puntaje total de la Escala Mexicana de Resiliencia. $\mathrm{FC}=$ Fortaleza y confianza en sí mismo. $\mathrm{CS}=$ Competencia social. $\mathrm{AF}=$ Apoyo familiar. $\mathrm{AS}=$ Apoyo social. $\mathrm{E}=\mathrm{Estructura}$. Variables de religiosidad: Convicción $=$ ¿En qué grado se considera creyente? Práctica $=$ ¿Con qué frecuencia sigue los rituales y ceremonias? Variables existenciales: Cambio $=i$ Su forma de ver la vida cambio durante el transcurso de la enfermedad? Sentido $=$ ¿Considera que esta situación, le dio mayor sentido a su vida? Variables clínicas: Tiempo = tiempo transcurrido en años desde el diagnóstico de cáncer. Efectos = efectos secundarios de los medicamentos o tratamientos ocasionan mas deterioro físico que la propia enfermedad, Información = información sobre la enfermedad, tratamiento y su proceso de enfermedad. Tratamiento = percepción de que el tratamiento está teniendo éxito. Adherencia $=$ seguir adecuadamente el tratamiento y las indicaciones del médico. 
TABLA 2.

Modelos de regresión estandarizados para predecir las respuestas psicológicas del duelo.

\begin{tabular}{|c|c|c|c|c|c|c|c|c|}
\hline \multirow{2}{*}{$\begin{array}{c}\text { Variables } \\
\text { predictoras }\end{array}$} & \multicolumn{8}{|c|}{ Variables predichas } \\
\hline & ANE & APE & Nega & Ira & P/Nego & F/E & Depre & Acept. \\
\hline \multicolumn{9}{|l|}{ Alexitimia } \\
\hline TAS-20 & $.41^{* * *}$ & & $.30^{\star * *}$ & $.31^{* * *}$ & & \multirow{2}{*}{\multicolumn{3}{|c|}{$.46^{\star \star \star}$}} \\
\hline DIS & & & & & & & & \\
\hline \multicolumn{9}{|l|}{ Resiliencia } \\
\hline RESI & & & & & & $.21^{*}$ & & $.38^{* * *}$ \\
\hline $\mathrm{AF}$ & $-.32^{\star * *}$ & & & $-.33^{* * *}$ & & & & \\
\hline $\mathrm{E}$ & $-.19^{*}$ & $.26^{\star \star}$ & & & & & & \\
\hline \multicolumn{9}{|c|}{ Variables sociodemográficas } \\
\hline Ingreso & & $-.36^{* * *}$ & $-.20^{*}$ & & $-.31^{\star \star *}$ & & & \\
\hline Escolaridad & & & & & & $-.33^{* * *}$ & & \\
\hline \multicolumn{9}{|c|}{ Variables de religiosidad } \\
\hline Convicción & & $.18^{*}$ & & & $.20^{*}$ & & & \\
\hline Práctica & & & & & & $.22^{*}$ & $-.18^{\star}$ & \\
\hline \multicolumn{9}{|l|}{ Variable existencial } \\
\hline Sentido & & & & & & $.19^{*}$ & & $.19^{*}$ \\
\hline \multicolumn{9}{|l|}{ Variables clínicas } \\
\hline Efectos & $.18^{*}$ & & & & & & $.25^{\star *}$ & \\
\hline Adherencia & & & & $-.20^{*}$ & & & & \\
\hline Información & & & & & & & $-.16^{\star}$ & $.17^{*}$ \\
\hline$\%$ var. explicada & $47 \%$ & $25 \%$ & $17 \%$ & $32 \%$ & $14 \%$ & $26 \%$ & $39 \%$ & $29 \%$ \\
\hline
\end{tabular}

Método: Pasos progresivos. Significación del coeficiente de determinación: ${ }^{*} p \leq .05,{ }^{* *} p \leq .01 \mathrm{y}{ }^{* * *} p \leq .001$. Respuestas psicológicas del duelo: ANE $=$ Afecto negativo ante la enfermedad. APE $=$ Actitud positiva ante la enfermedad. Nega $=$ Negación, P/Nego $=$ Promesas $/$ negociación, Fe/Es $=$ Fe/esperanza, Depre $=$ Depresión y Acep $=$ Aceptación. Alexitimia: TAS-20 = Puntuación total de la escala TAS-20. DIS $=$ Dificultad para identificar sentimientos. Resiliencia: RESI = Puntaje total de la Escala Mexicana de Resiliencia. AF = Apoyo familiar. E = Estructura. Variable existencial: Sentido $=$ ¿Considera que esta situación, le dio mayor sentido a su vida?. Variables de religiosidad: Convicción $=$ ¿En qué grado se considera creyente? Práctica = ¿Con qué frecuencia sigue los rituales y ceremonias?. Variables clínicas: Efectos = efectos secundarios de los medicamentos o tratamientos ocasionan mas deterioro físico que la propia enfermedad. Adherencia $=$ seguir adecuadamente el tratamiento y las indicaciones del médico e Información = información sobre la enfermedad, tratamiento y su proceso de enfermedad.

Especificación y contraste del modelo estructural de regresión

Como variables latentes se introdujeron las dos dimensiones de las respuestas psicológicas del duelo ante la pérdida de la salud. El modelo de medida de la variable latente de afecto negativo quedó configurado por 4 variables manifiestas: ira, depresión, aceptación y negación. El modelo de medida de la variable latente de actitud positiva ante la enfermedad quedó configurado por tres variables manifiestas: fe/esperanza, promesas/negociación y negación. Se escogieron como variables manifiestas exógenas a las 6 variables predictoras de los modelos de regresión de ANE y APE (véase Tabla 2). La variable latente endógena de bajo afecto negativo fue predicha por las variables manifiestas exógenas: alexitimia (puntuación total de la TAS-20), apoyo familiar, estructura y efectos secundarios del tratamiento oncológico. La variable latente endógena de actitud positiva ante la enfermedad fue predicha por las variables manifiestas exógenas: ingreso económico familiar, estructura y convicción religiosa. Se especificaron tres correlaciones entre las seis variables manifiestas exógenas: alexitimia con ingreso económico familiar y apoyo familiar, así como apoyo familiar con estructura. Todos los residuos de medida y los disturbios fueron independientes entre sí (véase Figura 1). 
Previo al cálculo del modelo estructural se hizo un análisis de correlación canónica para estimar la asociación lineal entre el conjunto de variables predictoras y el conjunto de respuestas psicológicas que configura cada factor de duelo. La correlación canónica entre el conjunto de variables predictoras (alexitimia 0 puntuación total de la TAS-20, apoyo familiar, estructura y efectos secundarios) y el conjunto de variables predichas de afecto negativo (ira, depresión, aceptación y negación) fue muy alta $\left(r_{c}=.83\right)$. La correlación canónica entre el conjunto de variables predictoras (estructura, ingresos económicos familiares y convicción en las creencias religiosas) y el conjunto de variables predichas de actitud positiva (fe/esperanza, promesas/negociación y negación) también fue muy alta $\left(r_{c}=.78\right)$.

Al estimar los parámetros, el coeficiente estructural y el tamaño del efecto directo de la convicción religiosa sobre la actitud positiva no fueron significativos tanto por ML como por el método de percentiles libres de sesgo; tampoco fueron significativos los tamaños del efecto indirecto de la convicción religiosa sobre los tres indicadores de actitud positiva.

\section{FIGURA 1.}

Modelo estandarizado inicial estimado por Máxima Verosimilitud.

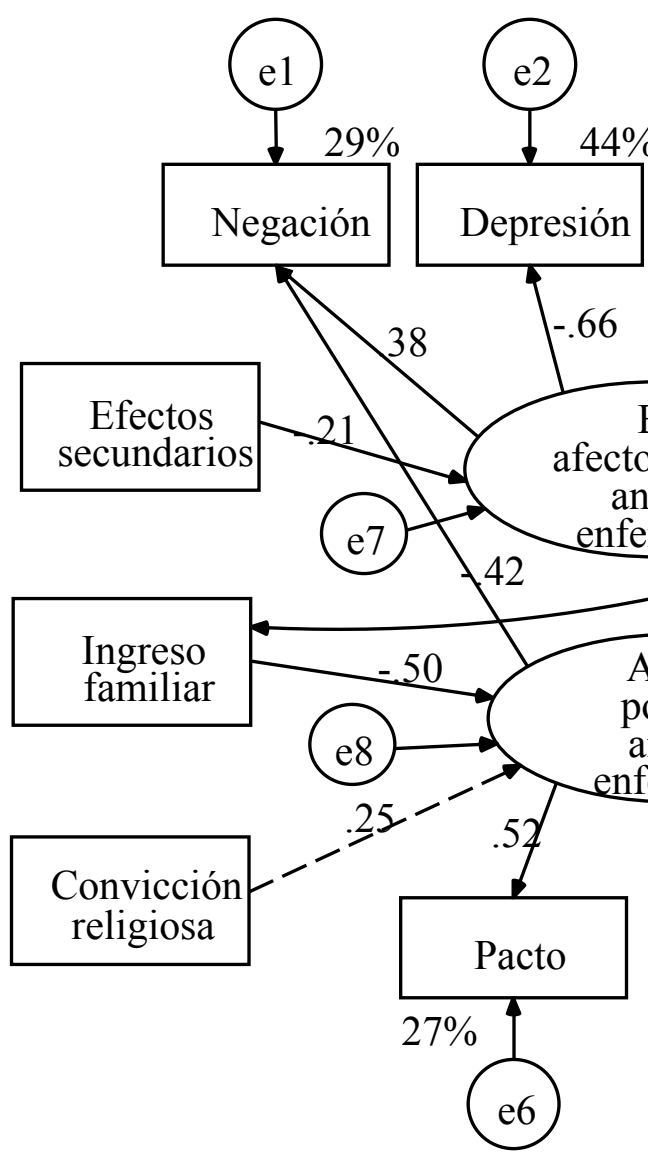

Tras eliminar convicción religiosa del modelo estructural, todos los parámetros fueron significativos tanto por la estimación $\mathrm{ML}$ como por el método de percentiles libres de sesgo (véase Figura 2). La bondad de ajuste se mantuvo por la probabilidad de muestreo repetitivo de Bollen-Stine $(p=$

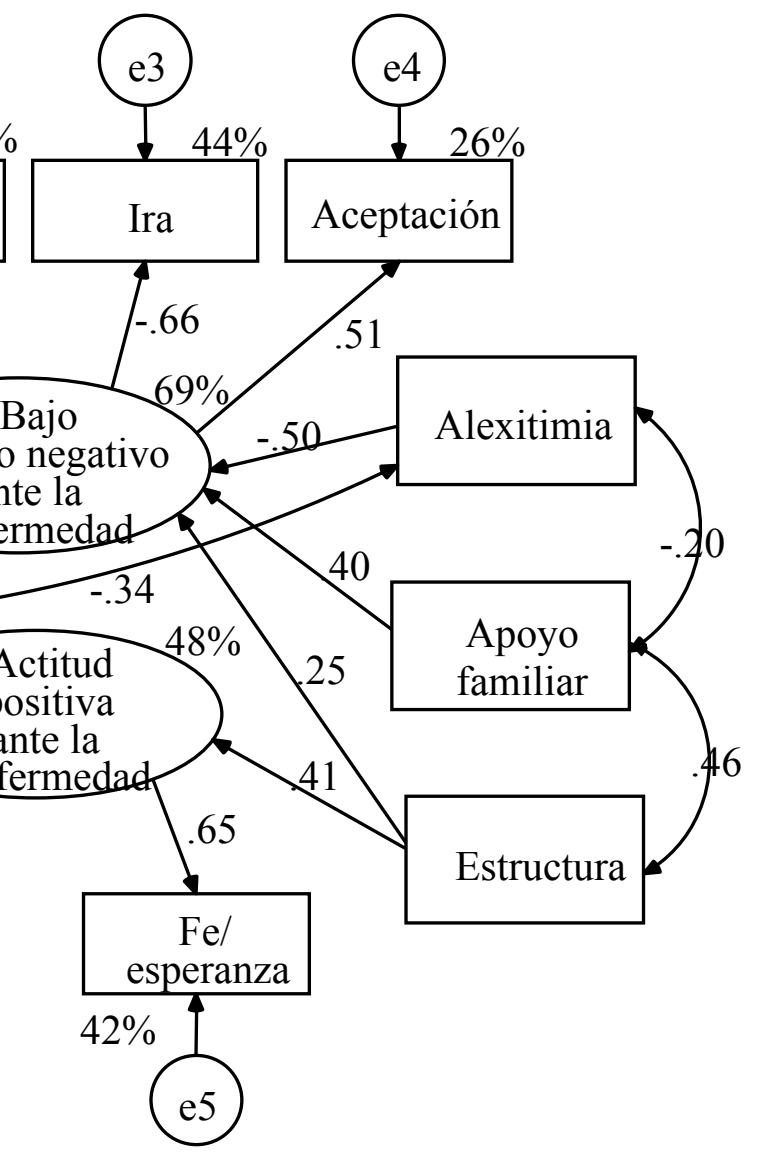

$1,008 / 2,000=.50)$. Los índices de ajuste variaron de buenos $(X 2[39, N=120]=$ $48.50, p=.14, x 2 / g \mid=1.24, C F I=.96 \mathrm{y}$ RMSEA $=.04)$ a adecuados (GFI $=.94$, $\mathrm{AGFI}=.89, \mathrm{NFI}=.84$ y RMS SR $=.07) . \mathrm{El}$ modelo tuvo parsimonia alta $(\mathrm{RP}=.71)$. 
FIGURA 2.

Modelo estandarizado revisado estimado por Máxima Verosimilitud.

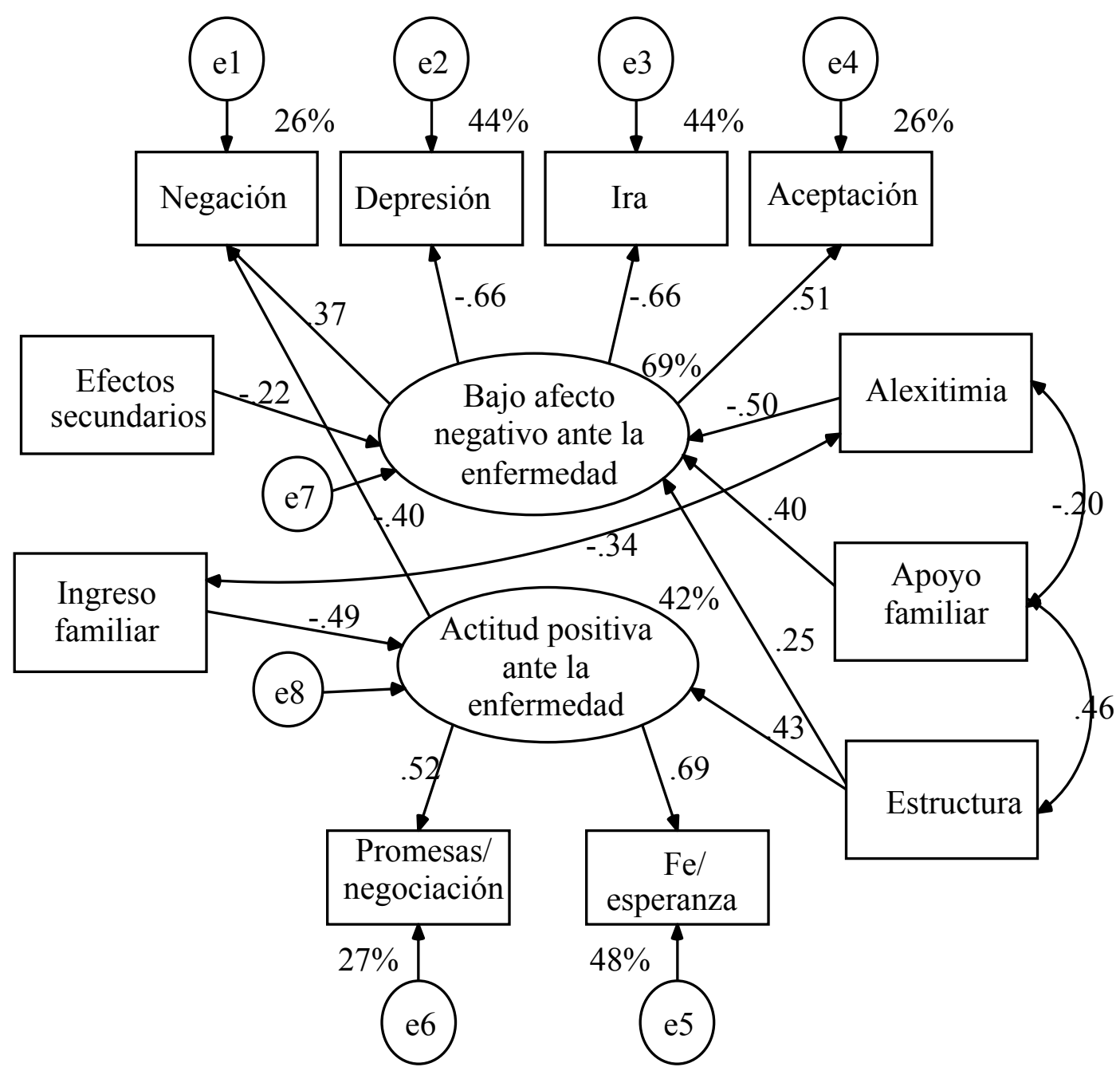

Dentro del modelo de medición del bajo afecto negativo, el efecto directo de esta variable latente fue grande $\mathrm{y}$ de signo negativo sobre ira y depresión; grande y de signo positivo sobre aceptación, y mediano y de signo positivo sobre negación. Mayor puntuación en bajo afecto negativo refleja menores respuestas de ira y depresión y mayores respuestas de aceptación y negación (véase Tabla 3). 
TABLA 3.

Tamaño del efecto y significación en los 2 modelos de medida y el modelo estructural.

\begin{tabular}{|c|c|c|c|c|c|c|c|c|}
\hline \multirow{2}{*}{$\begin{array}{l}\text { Variable } \\
\text { destino }\end{array}$} & \multirow[b]{2}{*}{ Efecto } & \multicolumn{7}{|c|}{ Variable de origen } \\
\hline & & $\begin{array}{l}\text { Apoyo } \\
\text { familiar }\end{array}$ & Estruc. & TAS20 & $\begin{array}{l}\text { Efectos } \\
\text { secund. }\end{array}$ & $\begin{array}{c}\text { Ingreso } \\
\text { económico }\end{array}$ & APE & ANE \\
\hline \multirow{2}{*}{$\begin{array}{l}\text { Actitud } \\
\text { positiva }\end{array}$} & $\mathrm{T}$ & & $.43^{* *}$ & & & $-.49^{* * *}$ & & \\
\hline & $\begin{array}{l}D \\
\text { I }\end{array}$ & & $.43^{\star *}$ & & & $-.49^{* * *}$ & & \\
\hline \multirow{2}{*}{$\begin{array}{c}\text { Bajo } \\
\text { afecto } \\
\text { negativo }\end{array}$} & $T$ & $.40^{* \star}$ & $.25^{\star}$ & $-.50^{* * *}$ & $-.22^{*}$ & & & \\
\hline & $\begin{array}{l}D \\
\text { I }\end{array}$ & $.40^{* *}$ & $.25^{*}$ & $-.50^{\star * *}$ & $-.22^{*}$ & & & \\
\hline \multirow{3}{*}{$\begin{array}{l}\text { Promesas y } \\
\text { negociación }\end{array}$} & $T$ & & $.22^{* * *}$ & & & $-.26^{\star * \star}$ & $.52^{\star \star}$ & \\
\hline & D & & & & & & $.52^{\star *}$ & \\
\hline & I & & $.22^{\star * *}$ & & & $-.26^{\star * *}$ & & \\
\hline \multirow{3}{*}{$\begin{array}{c}\text { Fe y } \\
\text { esperanza }\end{array}$} & $T$ & & $.29^{\star *}$ & & & $-.34^{\star * *}$ & $.69^{* \star}$ & \\
\hline & $D$ & & & & & & $.69^{\star *}$ & \\
\hline & I & & $.29^{* \star}$ & & & $-.34^{\star * *}$ & & \\
\hline \multirow{3}{*}{ Aceptación } & $T$ & $.20^{\star *}$ & $.13^{*}$ & $-.25^{\star \star *}$ & $-.11^{*}$ & & & $.51^{\star *}$ \\
\hline & D & & & & & & & $.51^{* *}$ \\
\hline & 1 & $.20^{* *}$ & $.13^{*}$ & $-.25^{\star * *}$ & $-.11^{*}$ & & & \\
\hline \multirow{3}{*}{ Ira } & $\mathrm{T}$ & $-.27^{* *}$ & $-.17^{\star}$ & $.33^{\star \star *}$ & $.14^{*}$ & & & $-.67^{* *}$ \\
\hline & D & & & & & & & $-.67^{* *}$ \\
\hline & I & $-.27^{\star \star}$ & $-.17^{\star}$ & $.33^{\star * *}$ & $.14^{*}$ & & & \\
\hline \multirow{3}{*}{ Depresión } & $\mathrm{T}$ & $-.27^{\star *}$ & $-.17^{\star}$ & $.33^{\star \star *}$ & $.14^{*}$ & & & $-.66^{\star *}$ \\
\hline & D & & & & & & & $-.66^{* *}$ \\
\hline & I & $-.27^{\star \star}$ & $-.17^{\star}$ & $.33^{\star \star *}$ & $.14^{*}$ & & & \\
\hline \multirow{3}{*}{ Negación } & $T$ & $.15^{\star \star}$ & $-.08 \mathrm{~ns}$ & $-.18^{* *}$ & $-.08^{*}$ & $.20^{* \star}$ & $-.40^{\star *}$ & $.37^{* *}$ \\
\hline & D & & & & & & $-.40^{\star *}$ & $.37^{* *}$ \\
\hline & I & $.15^{\star *}$ & $-.08 \mathrm{~ns}$ & $-.18^{* *}$ & $-.08^{*}$ & $.20^{* *}$ & & \\
\hline
\end{tabular}

Dentro del modelo de medición de la actitud positiva ante la enfermedad, el efecto directo de esta variable latente fue grande y de signo positivo sobre fe/esperanza y promesas/negociación, y mediano y de signo negativo sobre negación. Mayor puntuación en actitud positiva refleja mayores respuestas de fe/esperanza y promesas/negociación, así como menor negación (véase Tabla 3).

Apoyo familiar tuvo un efecto directo, mediano y de signo positivo sobre bajo afecto negativo. El efecto indirecto del apoyo familiar sobre los cuatro indicadores de bajo afecto negativo fue pequeño; de signo negativo sobre ira y depresión, y de signo positivo sobre aceptación y negación. Mayor apoyo familiar predice directamente bajo afecto negativo ante la enfermedad, e indirectamente menores respuestas de ira y depresión, y mayores respuestas de aceptación y negación (véase Tabla 3).

El efecto de estructura sobre las dos variables latentes fue directo y de signo positivo; su tamaño fue mediano sobre actitud positiva y pequeño sobre bajo afecto negativo. El efecto indirecto de estructura sobre los indicadores de estas dos variables latentes fue pequeño; de signo positivo sobre fe/esperanza, promesas/negociación y aceptación, y de signo negativo sobre ira y depresión. El efecto indirecto sobre negación no fue significativo. Mayor estructura predice directamente mayor actitud positiva y bajo afecto negativo, e indirectamente mayor fe/esperanza, mayor promesas/negociación, menor ira, menor depresión y mayor aceptación (véase Tabla $3)$. 
La alexitimia tuvo un efecto directo, grande y de signo negativo sobre bajo afecto negativo. En relación con los cuatro indicadores de bajo afecto negativo, el efecto indirecto de la alexitimia fue mediano y de signo positivo sobre ira y depresión, y fue pequeño y de signo negativo sobre aceptación y negación. Menor alexitimia predice directamente bajo afecto negativo, e indirectamente menor ira y depresión y mayor aceptación y negación (véase Tabla 3).

Efectos secundarios del tratamiento oncológico tuvo un efecto directo, pequeño y de signo negativo sobre bajo afecto negativo. En relación con los cuatro indicadores de bajo afecto negativo, el efecto indirecto de los efectos secundarios del tratamiento oncológico fue pequeño; de signo positivo sobre ira, depresión, y de signo negativo sobre aceptación y negación. Menos síntomas de efectos secundarios del tratamiento predicen directamente bajo afecto negativo, e indirectamente menor ira y depresión y mayor aceptación y negación (véase Tabla 3).

Ingreso económico familiar tuvo un efecto directo y mediano sobre actitud positiva. En relación con los dos indicadores de actitud positiva, el efecto indirecto del ingreso económico sobre fe/esperanza fue mediano y de signo negativo, sobre promesas/negociación fue pequeño y de signo negativo y sobre negación fue pequeño y de signo positivo. Menor ingreso económico predice directamente una actitud más positiva ante la enfermedad, e indirectamente más fe/esperanza, más promesas/negociación y menos negación (véase Tabla 3).

La alexitimia correlacionó significativamente y con signo negativo con ingreso económico familiar y apoyo familiar, siendo la magnitud de la asociación de la primera correlación mediana y de la segunda pequeña. A mayor alexitimia, menor ingreso familiar y menor apoyo familiar, y viceversa. El apoyo familiar correlacionó significativamente y con signo positivo con estructura, siendo la magnitud de la asociación mediana. A mayor apoyo familiar, mayor estructura y viceversa (véase Figura 2).

Se calculó la potencia del contraste del modelo estructural de regresión a partir del estadístico RMSEA. El nivel de significación se fijó en .05. Como hipótesis nula se tomó el valor medio del estadístico RMSEA del modelo especificado (.03) y como hipótesis alternativa el valor medio del estadístico RMSEA del modelo independiente (.18). Siendo los grados de libertad del modelo especificado 49 y el tamaño de la muestra de 120 participantes, la potencia fue unitaria.

\section{DISCUSIÓN}

Con respecto al objetivo de determinar los correlatos significativos de las respuestas psicológicas del duelo, como se esperaba, la alexitimia (puntuación total y sus tres factores), resiliencia (puntuación total y sus cinco factores), variables sociodemográficas (ingreso y escolaridad), religiosidad (práctica y convicción), existenciales (sentido) y clínicas (efectos secundarios, información, percepción de éxito del tratamiento y adherencia al tratamiento) se asociaron significativamente con las respuestas psicológicas del duelo, especialmente con la dimensión de bajo afecto negativo y las respuestas de depresión, aceptación e ira, alcanzando algunas de estas correlaciones magnitudes de asociación altas. También, conforme a las expectativas, las magnitudes de las asociaciones de alexitimia y resiliencia con las respuestas psicológicas del duelo fueron mayores que las magnitudes de las asociaciones de las variables sociodemográficas, de religiosidad, existenciales y clínicas, resultando la alexitimia la variable con correlaciones más altas. 
El tiempo transcurrido desde el diagnóstico al momento de la encuesta fue independiente de los factores y dimensiones de las respuestas psicológicas del duelo. Este resultado no debe atribuirse a una reducida variabilidad o limitación de valores en la variable tiempo, pues el rango fue de 1 mes a 13 años con una mediana y moda de 1 año y la unidad de medida fue en meses. Tampoco a una tamaño reducido de muestra. El resultado es congruente con el pobre apoyo empírico del modelo secuencial del duelo frente al modelo de respuestas que coexisten y se suceden en diverso orden (Holland \& Neimeyer, 2010; Isla, Moncho, Guasch, \& Torras, 2008) y con los resultados de otros estudios de aceptación de la enfermedad (Zalewska et al., 2007).

La edad también fue independiente. Aunque la edad ha resultado independiente de las respuestas psicológicas del duelo en otras investigaciones (Zalewska et al., 2007), algunos estudios han encontrado menos afecto negativo (Burgess et al., 2005), menos ira (Exline et al., 2011) y más aceptación (Thompson et al., 2009) entre pacientes femeninas con mayor edad, usando muestras más homogéneas en sus características sociodemográficas y clínicas que la presente muestra.

El cambio en la visión de la vida por padecer cáncer también fue independiente. Esto se podría atribuir a la vaguedad de la pregunta que provocó que la mayoría de mujeres indicaran que sí habían experimentado mucho cambio (dos tercios de ellas), ya sea que tuvieran 0 no complicaciones en su proceso del duelo. Una pregunta más específica en relación con lo positivo o negativo en este cambio, como la pregunta sobre el sentido de la vida, probablemente sí proporcione una correlación significativa. Las mujeres con un cambio negativo probablemente tengan más complicaciones en su proceso del duelo, especialmente si el cambio fue grande.
Con respecto al objetivo de determinar los predictores significativos de las respuestas psicológicas del duelo, la alexitimia (puntuación total y su factor de dificultad para identificar sentimientos), resiliencia (puntuación total y sus factores de estructura y apoyo familiar), las variables sociodemográficas de ingreso y escolaridad, religiosidad, la variable existencial de sentido y las variables clínicas de efectos secundarios del tratamiento, información y adherencia al tratamiento fueron predictores significativos. Además su efecto lineal resultó con el signo esperado. La alexitimia, bajos ingresos económicos, baja escolaridad, más efectos secundarios, baja adherencia al tratamiento y no poder dar un nuevo sentido a la vida ante la enfermedad actuaron como factores de riesgo, y resiliencia y religiosidad actuaron como factores protectores. También conforme a la expectativa el mayor peso fue de las variables de personalidad, siendo mayor el peso de la alexitimia que el de la resiliencia.

Se introdujo la puntuación total de la TAS-20 en el modelo estructural de regresión por tres motivos: 1) contar con una evaluación global del constructo de la alexitimia, 2) sus tres factores fueron correlatos significativos de una o las dos dimensiones del duelo y 3) la puntuación total fue la variable que entró en la mayoría de los modelos de regresión. En la presente muestra pensamiento externamente orientado tuvo una consistencia interna alta, cuando en otros estudios su consistencia es baja y usualmente no se correlaciona con los constructos estudiados y relevantes para la alexitimia (Taylor \& Bagby, 2013).

La alexitimia fue el predictor con mayor efecto sobre el afecto negativo ante la enfermedad, concordante con la expectativa (Mantani et al., 2007; Taylor \& Bagby, 2013). En este sentido, diversos estudios han señalado la importancia de ofrecer la oportunidad al paciente de expresar sus sentimientos con respecto a la enfermedad y compartir con otras personas la carga 
emocional que están experimentado para lograr un proceso exitoso de duelo (Kreicbergs, Lannen, Onelov \& Wolfe, 2007; Worden, 2010). Una manera de llevar a cabo el reconocimiento de las emociones y la expresión del dolor de la pérdida es mediante la terapia narrativa de Neimeyer (2000), la cual favorece la expresión de las emociones y la reconstrucción de significados tras la ocurrencia de la pérdida. No obstante, existen estudios que señalan que expresar las emociones en situaciones de duelo no siempre conlleva efectos positivos (Hooghe, Neimeyer \& Rober, 2011), incluso algunos señalan que el compartir las emociones puede intensificar el malestar emocional e interferir en el afrontamiento activo (Kennedy-Moore \& Watson, 1999). En este sentido, Coifman, Bonanno, Ray y Gross (2007) hallaron que el expresar las emociones negativas cuando la persona se está recuperando de un acontecimiento adverso no facilita la capacidad de recuperación, sino que el desviar la atención de la experiencia adversa facilita la elaboración del duelo. De todos estos hallazgos se colige que la rumiación del afecto negativo es contraproducente. La expresión de afecto sí puede tener un efecto positivo sobre el proceso de duelo si hay una reconstrucción del significado, el enfoque se pone sobre el afecto positivo y se evita la rumiación del afecto negativo (Neimeyer, 2000). Para movilizar estos recursos se requiere contar o desarrollar competencia emocional, esto es, bajo nivel de alexitimia y alta resiliencia. Los resultados del presente estudio prestan apoyo empírico al efecto protector de un bajo nivel de alexitimia y alto de resiliencia cuyo mecanismo de acción probablemente sea contar con los recursos para resignificación, focalización en lo positivo y evitación de la rumiación de lo negativo.

La alexitimia correlacionó con menor apoyo familiar y menor ingreso económico familiar en el modelo estructural especificado. La correlación negativa entre alexitimia y apoyo familiar confirma que las personas alexitímicas tienen dificultades para relacionarse con otras personas, incluyendo la familia, lo que afecta al apoyo familiar (Bratis et al., 2009). Asimismo, se presta apoyo empírico a los resultados de otras investigaciones que reportan una asociación significativa y de signo negativo entre alexitimia y estatus socioeconómico (Kokkonen et al., 2001). En este estudio la asociación fue moderada. Las participantes con menor ingreso económico familiar reportaron mayor nivel de alexitimia, probablemente mostrando mayores dificultades para identificar, verbalizar y considerar el afecto en la comunicación social y toma consciente de decisiones en comparación con las mujeres con mayor ingreso económico familiar. Este hallazgo también es concordante con estudios cualitativos de comunicación y clase social en Latinoamérica (Crespo \& Rojas, 2010).

Entre los factores de resiliencia, el apoyo familiar destacó como protector de menos afecto negativo ante la enfermedad. En este sentido Cardoso et al. (2012) mencionan que cuando se atraviesa por momentos de gran incertidumbre, el apoyo y la cohesión familiar aportan grandes beneficios a nivel físico y mental, como lo es una mejor recuperación. Por lo tanto, este resultado confirma que más que el apoyo social, es el apoyo familiar el que aminora las emociones negativas y es un factor protector importante en el proceso del duelo (Barreto et al., 2012; Blanchard, Albrecht, Ruckdeschel, Grant, \& Hemmick, 1995). No obstante, los presentes datos no prestan apoyo empírico a la propuesta de que la relación entre efectos secundarios y afecto negativo está mediada por el apoyo familiar (Blanchard et al., 1995), ya que apoyo familiar y efectos secundarios (variables predictoras de afecto negativo) fueron independientes, como se puede observar en el modelo estructural con buen ajuste a los datos. Entre los factores de resiliencia también destacó la estructura. Las mujeres que logran mantener el orden en sus vidas tienen tanto una actitud más positiva como menos afecto negativo, lo que 
se ve favorecido por el apoyo de la familia y, al contrario que la alexitimia, favorece que la familia apoye a la mujer.

La variable de efectos secundarios destacó como predictor entre las variables clínicas de los tratamientos oncológicos, generando más depresión y afecto negativo ante la enfermedad. En otros estudios con pacientes con cáncer se encontró que el dolor físico y la presencia de síntomas eran predictores de mayores síntomas de depresión (Gil et al., 2008) e ira (Remor, Amorós, \& Carrobles, 2006; Schlatter \& Cameron, 2010). Barreto et al. (2012) hallaron que los síntomas sin control durante todo el proceso de la enfermedad del paciente con cáncer predecían el duelo complicado en los deudos del paciente. No obstante, en este estudio, no es la percepción del efecto curativo, sino los síntomas secundarios lo que afecta al duelo, probablemente porque estos son más salientes y limitantes en la vida de estas mujeres en tratamiento oncológico.

Entre las variables sociodemográficas claramente destacó el ingreso económico con efecto mayor que el esperado. Tuvo un efecto moderado sobre actitud positiva ante la enfermedad en el modelo estructural y fue predictor de negación $y$ promesas/negociación. Esto puede atribuirse a las desigualdades de salud en México (Aguilera et al., 2005). Las mujeres con menor ingreso económico familiar, usualmente con trabajos sin contrato, se enfrentan a una asistencia sanitaria llena de insuficiencias y demoras. Así, parecen buscar refugio en la fe religiosa para compensar la adversidad derivada de los obstáculos para acceder a los procesos diagnósticos, mantener el tratamiento y solventar las cargas económicas que conllevan la atención clínica y su incapacidad para trabajar.

En el modelo estructural de regresión inicialmente especificado se incluyó convicción religiosa por ser predictor en el modelo de regresión para predecir afecto positivo, pero no tuvo un peso significativo y se eliminó en el modelo con buen ajuste. El mismo resultado de falta de significación se tendría si en lugar de introducir convicción se hubiera introducido práctica religiosa. Por lo tanto, se presta apoyo empírico a la asociación significativa y poder predictivo de la religiosidad en tres respuestas y una dimensión del duelo (Exline et al. 2011), pero las dos variables de religiosidad poseen un peso pequeño y finalmente espurio ante las otras variables en el modelo integrador final, sobre todo ante los constructos de personalidad y el ingreso económico familiar.

Como limitaciones del estudio debe mencionarse que el diseño fue no experimental transversal, de ahí que no se pueden hacer inferencias de causalidad y sólo se habla de correlatos y predictores. Otra de las limitaciones fue el carecer de una muestra poblacional, habiéndose realizado en una muestra incidental de mujeres con cáncer bajo tratamiento oncológico, por lo que los resultados deben manejarse como hipótesis para futuras investigaciones en esta población y otras afines. Inicialmente se pretendía obtener una muestra de al menos 200 participantes, la mitad mujeres y la mitad hombres. Sin embargo, resultó imposible obtener una muestra de 100 hombres, debido a que en los centros en los cuales se aplicaron los cuestionarios y en los cuales se contaba con permiso de las autoridades, acudían mayoritariamente mujeres. A pesar del tamaño de muestra limitado, se alcanzó una potencia unitaria desde el estadístico RMSEA en el contraste del modelo estructural y la mayoría de los efectos directos fueron altos sobre las variables endógenas, lo que disminuye este efecto en la estimación de parámetros y contraste del ajuste. Se podrían alegar problemas por incumplimiento de normalidad multivariada; no obstante, se acudió a los procedimientos de muestreo repetitivo con la extracción de 
un número grande de muestras (Kline, 2010).

En conclusión, en la presente muestra de mujeres mexicanas tratadas de cáncer, alexitimia, resiliencia, las variables sociodemográficas de ingreso y escolaridad, religiosidad, la variable existencial de sentido de la vida y las variables clínicas de efectos secundarios del tratamiento, información, percepción del efecto curativo del tratamiento y adherencia al tratamiento se asociaron con las respuestas psicológicas del duelo. En el modelo integrador, bajo afecto negativo ante la enfermedad, caracterizado por menores reacciones de ira y depresión y mayor aceptación y negación, fue predicho por menor alexitimia, mayor apoyo familiar, mayor estructura y menores efectos secundarios del tratamiento. La actitud positiva ante la enfermedad caracterizada por mayores promesas/negociación con un Ser Supremo y mayor fe/esperanza en la recuperación fue predicha por mayor estructura y menor ingreso económico familiar. La alexitimia tuvo el mayor peso significativo al predecir bajo afecto negativo y se asoció con menor apoyo familiar y menor ingreso económico familiar; le siguió el menor apoyo familiar que se asoció, a su vez, con menor estructura en la vida de la paciente. El aspecto de la resiliencia de continuar manteniendo la rutina diaria aún en momentos difíciles fue un factor protector de una mayor actitud positiva y menor afecto negativo ante la enfermedad. Además, se encontró que menor ingreso económico familiar predice la actitud positiva ante la enfermedad (basada en creencias religiosas) con mayor peso que estructura, probablemente debido al fuerte apoyo en la fe religiosa ante las desigualdades de salud que viven muchas de estas mujeres.

Se sugiere trabajar en los recursos cognitivo-emocionales en pacientes con rasgos de alexitimia, lo que hará más probable concluir con éxito el proceso del duelo ante la pérdida de salud. Se recomienda incluir dentro de las intervenciones psicológicas el fortalecimiento de la red de apoyo familiar, especialmente en pacientes alexitímicas que probablemente requerirán entrenamiento en habilidades sociales. Se sugiere poner énfasis en las metas prioritarias que tienen las pacientes, su manera de planeación a corto, mediano y largo plazo, con la finalidad de que la enfermedad no realice cambios trascendentales en su estilo de vida o sea un obstáculo que le impida continuar haciendo aquellas actividades, proyectos 0 roles que le dan sentido a su vida. También se insiste en que los profesionales de la salud estén atentos y monitoreen los efectos secundarios que los tratamientos médicos estén produciendo en las pacientes. Finalmente se insta a las autoridades sanitarias a que sigan trabajando en los problemas de desigualdad en salud para que las mujeres de bajos recursos no tengan que encomendarse sólo a la voluntad de Dios ante las negativas e insuficiencias de la atención clínica. 
REFERENCIAS

Aguilera, N., Marrufo, G. M., \& Montesinos, A. (2005). Desigualdad en salud en México: un análisis de sus determinantes. Ciudad de México: Universidad Iberoamericana.

American Psychological Association (2002). Ethical principles of psychologists and code of conduct. American Psychologist, 57, 1060-1073. doi:10.1037/0003-066X.57.12.1060.

Barreto, P., de la Torre, O., \& Pérez, M. (2012). Detección de duelo complicado. Psicooncología, 9, 355-368. doi: 10.5209/rev_PSIC.2013.v9.n2-3.40902.

Blanchard, C. G., Albrecht, T. L., Ruckdeschel, J. C., Grant, C. H., \& Hemmick, R. M. (1995). The role of social support in adaptation to cancer and to survival. Journal of Psychosocial Oncology, 13, 75-95. doi:10.1300/J077V13N01_05

Bratis, D., Tselebis, A., Sikaras, C., Moulou, A., Giotakis, K., Zoumakis, E., \& llias, I (2009). Alexithymia and its association with burnout, depression and family support among Greek nursing staff. Human Resources for Health, 7(72). Disponible en http://www.ncbi.nlm.nih.gov/pmc/article s/PMC2730051/pdf/1478-4491-7-72.pdf doi:10.1186/1478-4491-7-72.

Bonanno, G. A. (2004). Loss, trauma, and human resilience: have we underestimated the human capacity to thrive after extremely aversive events? American Psychologist, 59, 20-28. doi: 10.1037/0003-066X.59.1.20.

Burgess, C., Cornelius, V., Love, S., Graham, J., Richards, M., \& Ramirez, A. (2005). Depression and anxiety in women with early breast cancer: five year observational cohort study. British Medical Journal, 330, 702-705. Disponible en: http://www.bmj.com/content/330/7493/7 02.

Büssing, A., \& Koenig, H. G. (2010). Spiritual needs of patients with chronic diseases. Religions, 1, 18-27. doi:10.3390/rel1010018.

Cardoso, M. J., Ramos, M. I., Vaz, F. J., Rodríguez, L., \& Fernández, N. (2012). Influencia del apoyo familiar en momentos de gran incertidumbre. Revista Prisma Social, 8, 28-47.

Coifman, K. G., Bonanno, G. A., Ray, R. D., \& Gross, J. J. (2007). Does repressive coping promote resilience? Affectiveautonomic response discrepancy during bereavement. Journal of Personality and Social Psychology, 92(4), 745-758. doi: 10.1037/00223514.92.4.745

Crespo, N., \& Rojas, D. (2010). Clase social y desarrollo de la conciencia metapragmática de los niños. Estudios filológicos, 46, 25-41. Recuperado de http://www.scielo.cl/scielo.php?script=s ci_arttext\&pid=S0071-

$17132010000200002 \&$ lng=es\&tlng=es. doi:10.4067/S0071-

17132010000200002.

Exline, J. J., Park, C. L., Smyth, J. M., \& Carey, M. P. (2011). Anger toward God: social-cognitive predictors, prevalence, and links with adjustment to bereavement and cancer. Journal of Personality and Social Psychology, 100, 129-148. doi: 10.1037/a0021716.

Gil, F. L., Costa, G., Pérez, F. J., Salamero, M., Sánchez, N., \& Sirgo, A. (2008). Adaptación psicológica y prevalencia de trastornos mentales en pacientes con cáncer. Medicina Clínica, 130(3), 90-92.

Grossarth-Maticek, R., \& Eysenck, H. J. (1991). Creative novation behaviour therapy as a prophylactic treatment for cancer and coronary heart disease: Part l--Description of treatment. Behavior Research Therapy, 29, 1-16.

Hirsch, M. (2010). Coping with grief and loss: A guide to healing. Boston, MA: Harvard Health Publications.

Holland, J. M., \& Neimeyer, R. A. (2010). An examination of stage theory of grief among individuals bereaved by natural and violent causes: a meaning-oriented 
contribution. Journal of Death and Dying, $\quad 61, \quad$ 103-120. doi:10.2190/OM.61.2.b

Hooghe, A., Neimeyer, R. A., \& Rober, P. (2011). The complexity of couple communication in bereavement: an illustrative case study. Death Studies, 35(10), 905-924. doi: 10.1080/07481187.2011.553335

Isla, P., Moncho, J., Guasch, O., \& Torras, A. (2008). Proceso de adaptación a la diabetes mellitus tipo 1 (DM1). Concordancia con las etapas del proceso de duelo descrito por KüblerRoss. Endocrinología y Nutrición, 55(2), 78-83.

Kennedy-Moore, E., \& Watson, J. C. (1999). Expressing emotion: Myths, realities, and therapeutic strategies. New York, NY: Guilford.

Kline, R. B. (2010). Principles and practice of structural equation modeling ( $3^{\mathrm{a}} \mathrm{ed}$.), New York, NY: The Guilford Press.

Kokkonen, P., Karvonen, J. T., Veijola, J., Läksy, K., Jokelainen, J., Järvelin, M. R., \& Joukamaa, M. (2001). Prevalence and sociodemographic correlates of alexithymia in a population sample of young adults. Comprehensive Psychiatry, 42, 471-476. doi:10.1053/comp.2001.27892

Kübler-Ross, E. (2005). On grief and grieving: finding the meaning of grief through the five stages of loss. New York, NY: Simon \& Schuster.

Kreicbergs, U. C., Lannen, P., Onelov, E., \& Wolfe, J. (2007). Parental grief after losing a child to cancer: impact of professional and social support on long-term outcomes. Journal of the American Society of Clinical Oncology, 25, 3307-3312. doi: 10.1200/JCO.2006.10.0743JCO.

Lumley, M. A. (2004). Alexithymia, emotional disclosure, and health: a program of research. Journal of Personality, 72, 1271-1300. doi: 10.1111/j.1467-6494.2004.00297.x

Mantani, T., Saeki, T., Inoue, S., Okamura, H., Daino, M., Kataoka,T., \& Yamawaki,
S. (2007). Factors related to anxiety and depression in women with breast cancer and their husbands: role of alexithymia and family functioning. Supportive Care in Cancer, 15, 859868. doi: 10.1007/s00520-006-0209-4.

Miaja, M., \& Moral, J. (2014). Desarrollo y validación de la Escala de Respuestas Psicológicas de Duelo ante la Pérdida de la Salud (RPD-PS-38). Revista Psico-Oncología, 11, 369-387. doi: 10.5209/rev_PSIC.2014.v11.n23.47395

Moral, J. (2008). Propiedades psicométricas de la Escala de Alexitimia de Toronto de 20 reactivos en México. Revista Electrónica de Psicología Iztacala. 11(2), 97-114.

Neimeyer, R. A. (2000). Lessons of loss. Florida: PsychoEducational Resources.

O'Brien, C. W., \& Moorey, S. (2010). Outlook and adaptation in advanced cancer: a systematic review. Psychooncology, 19, 1239-1249. doi: 10.1002/pon.1704.

Palomar, J., \& Gómez, N. E. (2010). Desarrollo de una escala de medición de la resiliencia con mexicanos (RESIM). Interdisciplinaria, 27(1), 7-22.

Pentz, M. (2005). Resilience among older adults with cancer and the importance of social support and spirituality-faith. Journal of Gerontological Social Work, 44(3), 3-22. doi:10.1300/J083v44n03 02.

Peteet, J. R., \& Balboni, M. J. (2013). Spirituality and religion in oncology. $C A$ : A Cancer Journal for Clinicians, 63, 280-289. doi: 10.3322/caac.21187

Remor, E., Amorós, M., \& Carrobles, J. A. (2006). El optimismo y la experiencia de ira en relación con el malestar físico. Anales de Psicología, 22, 37-44.

Rueda, B., \& Pérez, A. M. (2007). Estudio de la alexitimia y de los procesos emocionales negativos en el ámbito de los factores de riesgo y la sintomatología cardiovascular. Revista de Psicopatología y Psicología Clínica, $12,105-116$. 
Schlatter, M. C., \& Cameron, L. D. (2010). Emotional suppression tendencies as predictors of symptoms, mood, and coping appraisals during AC chemotherapy for breast cancer treatment. Annals of Behavioral Medicine, 40, 15-29. doi:10.1007/s12160-010-9204-6

Taylor, G. J. A., \& Bagby, R. M. (2013). Psychoanalysis and empirical research: The example of alexithymia. Journal of the American Psychoanalytic Association, 61, 99-133. doi:10.1177/0003065112474066

Thompson, G. N., Chochinov, H. M., Wilson, K. G., McPherson, C. J., Chary, S., O'Shea, F. M., Kuhl, D. R., Fainsinger, R. L., Gagnon, P. R., \& Macmillan, K. A. (2009). Prognostic acceptance and the well-Being of patients receiving palliative care for cancer. Journal of Clinical Oncology, 27, 5757-5762. doi: 10.1200/JCO.2009.22.9799.

Van Damme, S., Crombez, G., Van Houdenhove, B., Mariman, A., \& Michielsen, W. (2006). Well-being in patients with chronic fatigue syndrome: The role of acceptance. Journal of Psychosomatic Research, 61, 595-599. doi:10.1016/j.jpsychores.2006.04.015

Worden, J. W. (2010). El tratamiento del duelo: asesoramiento psicológico y terapia (Segunda edición). Barcelona: Paidós.

Zalewska, A., Miniszewska, J., Chodkiewicz, J., \& Narbutt, J. (2007). Acceptance of chronic illness in psoriasis vulgaris patients. Journal of the European Academy of Dermatology Venereology, 21, 235-242. doi:10.1111/j.14683083.2006.01912.x 